\title{
Autonomous Obstacle Avoidance Using Visual Fixation and Looming
}

Kunal Joarder

Daniel Raviv

Forida Atlantic University The Robotics Center \& The Electrical Engineering Department Boca Raton, FL 33431 and

Sensory Intelligence Group Robot Systems Division

U.S. DEPARTMENT OF COMMERCE Technology Administration National Institute of Standards and Technology Robot Systems Division Bldg. 220 Rm B124

Gaithersburg, MD 20899 



\section{Autonomous Obstacle Avoidance Using Visual Fixation and Looming}

Kunal Joarder
Daniel Raviv

Forida Atlantic University The Robotics Center \& The Electrical Engineering Department Boca Raton, FL 33431

and

Sensory Intelligence Group

Robot Systems Division

U.S. DEPARTMENT OF COMMERCE Technology Administration National Institute of Standards and Technology

Robot Systems Division

Bldg. 220 Rm B124

Gaithersburg, MD 20899

December 1992

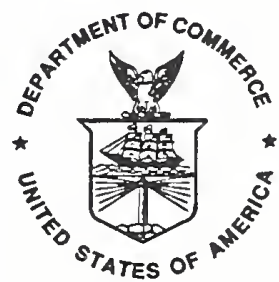

U.S. DEPARTMENT OF COMMERCE Barbara Hackman Franklin, Secretary

TECHNOLOGY ADMINISTRATION

Robert M. White, Under Secretary for Technology

NATIONAL INSTITUTE OF STANDARDS

AND TECHNOLOGY

John W. Lyons, Director 



\title{
AUTONOMOUS OBSTACLE AVOIDANCE USING VISUAL FIXATION AND LOOMING
}

\author{
Kunal Joarder and Daniel Raviv
}

Robotics Center and Department of Electrical Engineering

Florida Atlantic University

Boca Raton, Florida 33431; and

Robot Systems Division

National Institute of Standards and Technology (NIST)

Bldg. 220, Room B124, Gaithersburg, MD 20899 


\begin{abstract}
This paper describes a vision-based method for avoiding obstacles using the concepts of visual looming and fixating motion. Visual looming refers to the expansion of images of objects in the retina. Usually, this is due to the decreasing distance between the observer and the object. An increasing looming value signifies an increasing threat of collision with the object. The visual task of avoiding collision can be further simplified by purposive control of visual fixation at the objects in front of the moving camera. Using these two basic concepts real time obstacle avoidance in a tight perception-action loop is implemented. 3D space in front of the camera is divided into zones with various degrees of looming-based threat of collision. For each obstacle seen by a fixating camera, looming and its time derivative are calculated directly from the 2D image. Depending on the threat posed by an obstacle, a course change is dictated. This looming based approach is simple, independent of the size of the $3 \mathrm{D}$ object and its range and involves simple quantitative measurements. Results pertinent to a camera on a robot arm navigating between obstacles are presented.
\end{abstract}

Index Terms - Looming, Fixation, Visual Threat, Obstacle Avoidance. 



\section{INTRODUCTION}

How do we navigate in a world full of stationary and moving obstacles? How do we avoid collisions with furniture and moving people when we walk from one end of the room to the other ? How does a driver manipulate his speed and the course of direction during driving a car in a heavy traffic?

We argue that the visual looming effect, namely the expansion of objects' size in the retina is essential to all the above. Usually, an object's size as projected on the retina expands if the distance between the observer and the object decreases. This visual looming carries an indication of a possible collision with that object. The observer may react defensively to reduce this visual threat. Following this approach it is possible to explore navigation problems, in particular the obstacle avoidance problem, for an autonomous mobile robot. For navigation, reconstruction of 3D world is computationally expensive and may not be needed. In many situations a task-dependent, tight 2D based perception-action loop can be adopted [1-4].

The problem of obstacle avoidance by autonomous mobile robots has received various approaches from researchers in the computer vision area. Usually the task is to drive the robot from a starting position to a destination through multiple obstacles. In some cases an on-board sensor is not used and the environment is assumed to be known. An approach of global optimization to generate a trajectory as proposed by Gilbert [5], Shin [6], and Kim and Shin [7] requires intensive computation. Introduction of artificial potential fields that attract the robot to the goal and repulse the robot from obstacles has been proposed by Khatib [8], Hogan [9], Krogh [10,11], Newman and Hogan [12] and Volpe and Khosla [13]. However it is difficult to implement this method in the conventionally steered mobile robots. Nelson and Aloimonos [14] introduced an alternative, in which the flow field divergence is used as a qualitative measurement for obstacle avoidance.

In this paper, we use the visual parameter looming in servoing a camera attached to the end-effector of a six-degree-of-freedom robot through multiple spherical obstacles. It is shown that looming, a vital visual cue, when used actively by continuously changing the points of attention, can make it possible to navigate in a fairly complex environment. Our approach does not assume any knowledge on the velocity of the camera and the size and range of the obstacle. Looming is a measurable parameter that can be calculated directly from a sequence of 2D images obtained from an on-board camera. Hence the idea is simple and readily implementable.

First, we provide a summary of the previous results on looming, including definition and methods of measuring it. Visual fixation and looming as a function of time is covered next. Finally, the obstacle avoidance algorithm and its implementation are discussed and relevant results are presented. 


\section{LOOMING : SUMMARY OF PREVIOUS RESULTS}

Looming deals with expansion of the object's image on the retina. Usually this expansion is caused by a decreasing distance over some period of time between the observer and the object. Looming has been studied mostly qualitatively by the psychologists [15-19]. Recently a quantitative approach to looming has been presented by Raviv[20,21]. The looming value of an object is proportional to the relative translational velocity between the observer and the object divided by the distance itself. This measurable variate can be extracted directly from a sequence of 2-D images using optical flow or relative change in area of the image of the object. The concept of looming is also similar to the so called Nelson and Aloimonos' flow field divergence [14].

\subsection{Mathematical Definition of Looming:}

The looming value $\mathrm{L}$ of a very small $3 \mathrm{D}$ object is defined as the negative value of the time derivative of the relative distance between the observer and the center of the object divided by the relative distance[21]:

$$
L=-\frac{\frac{d R}{d t}}{R}
$$

The negative sign is used to signify image expansion with positive looming. The unit by which $\mathrm{L}$ is measured is $\left[\mathrm{time}^{-1}\right]$.

\subsection{Equal Looming Surfaces:}

Are there points in 3-D space that result in the same looming value $\mathrm{L}$ for any motion of the camera? It has been shown $[20,21]$ that such points for a particular looming value lie on a sphere. The center of the sphere is located on the instantaneous translation vector and the observer is located on a point on this sphere. A larger translational vector will produce a larger sphere. In our approach objects lying on an Equal Looming Sphere are considered to pose the same threat since they share the same looming value.

\subsection{Positive, Negative and Zero Looming Surfaces:}

Some of these equal looming surfaces correspond to positive values of looming, some correspond to negative values of looming and there is a plane (i.e., a sphere with infinite diameter) that correspond to zero value of looming. Figure 1 shows the three types of surfaces. The plane that passes through the pinhole point of the camera and is perpendicular to the instantaneous translational vector contains points that produce zero looming. Points on the hemisphere in front of the moving camera, (i.e., in front of the zero looming plane) produce 
positive values of looming and likewise points on the hemisphere behind the moving camera, (i.e., behind the zero looming plane) produce negative looming values. Naturally, objects lying on the zero or negative looming surfaces do not carry any threat of collision.

\subsection{Measurement of Looming Using Optical Flow :}

Using the well-known optical flow constraint equation [22], an expression of looming has been derived for a general six-degree-of-freedom motion of a camera [21].

Assume a stationary environment, with a spherical coordinate system $(\mathrm{R}, \theta, \phi)$ attached to a moving camera (Figure 2). Let the instantaneous angular velocity vector $\omega$ be represented as $(\mathrm{A}, \mathrm{B}, \mathrm{C})^{\mathrm{T}}$ where $\mathrm{T}$ denotes the transpose. Let $\mathrm{s}_{\theta}=\sin \theta, \mathrm{c}_{\theta}=\cos \theta, \mathrm{s}_{\phi}=\sin \phi, \mathrm{c}_{\phi}=\cos \phi$ and $\mathrm{I}_{\theta}, \mathrm{I}_{\phi}$ and $\mathrm{I}_{\mathrm{t}}$ are partial derivatives of the brightness I with respect to $\theta, \phi$ and time respectively. Then, the expression for looming can be written as :

$$
L=\frac{\left(c_{\phi_{r}} c_{\theta_{r}} c_{\phi} c_{\theta}+c_{\phi_{r}} s_{\theta_{r}} c_{\phi} s_{\theta}+s_{\phi_{t}} s_{\phi}\right)\left(-c_{\phi} I_{t}-c_{\phi}\left(B c_{\theta}-A s_{\theta}\right) I_{\phi}+\left(C c_{\phi}-s_{\phi}\left(B s_{\theta}+A c_{\theta}\right)\right) I_{\theta}\right)}{\left(c_{\phi_{1}} c_{r} s_{\theta}-c_{\phi_{1}} s_{\theta} c_{\theta}\right) I_{\theta}+c_{\phi}\left(c_{\phi_{r}} c_{\theta} s_{r} c_{\phi}+c_{\phi_{r}} s_{\theta} s_{\phi} s_{\theta}-s_{\phi_{t}} c_{\phi}\right) I_{\phi}}
$$

where the translation vector $\mathbf{t}$ forms $\theta=\theta_{\mathrm{t}}$ and $\phi=\phi_{\mathrm{t}}$ angles in the $\mathrm{R}-\theta-\phi$ coordinates.

Hence, given the location of the pixel in the image, the instantaneous rotational direction of motion, the direction of the instantaneous translational vector and the spatial and temporal intensity changes, the corresponding looming value of the corresponding point in $3 \mathrm{D}$ can be obtained.

\subsection{Measurement of Looming from Relative Rate of Expansion :}

The relative rate of expansion of a small object in the image is proportional to its looming value. This concept, as discussed in detail in [21], shows that the looming can be calculated from the projected area of a 3D object as :

$$
L=-\frac{\frac{d R}{d t}}{R} \approx \frac{\frac{d A}{d t}}{2 A}
$$

where $\mathrm{A}$ is the projected area of a 3D ball on a spherical image and $\mathrm{R}$ is the range of the ball from the camera. This idea of relative change of the projected area is used in our approach 
concerning obstacle avoidance strategy.

\section{VISUAL FIXATION AND LOOMING - AN OVERVIEW}

\subsection{Fixation :}

Visual fixation is actively manipulating the imaging system by directing the attention to some specific points. These points in 3D space carry a pertinent relationship with the task being performed. The fixation point may be stationary or in motion with the imaging system. By changing fixation or the point of attention an autonomous system can reduce a vast $3 \mathrm{D}$ space into a small working domain and hence by reducing the computational complexity it can simplify the visual tasks involved. Fixation obviates a very high resolution wide field of view imaging system. Moreover, fixation and a logarithmic retina simplifies the calculation of looming $[20,21]$. The relevant concept is motivated by the well known process in which alternate saccadic eye movement and fixation are witnessed in human obstacle avoidance [23].

\subsection{Looming and Changing Fixation as a Function of Time:}

It has already been mentioned that equal looming surfaces are spheres. The size of each sphere is proportional to the instantaneous translational velocity. As a camera approaches a point, the equal looming spheres containing this point shrink, producing higher values of looming. The centers of the spheres lie on a line along the instantaneous velocity vector (not necessarily on the optical axis) and the observer is located on a point on the sphere surface. Also it has been shown that the spheres are independent of the instantaneous rotational parameters $[20,21]$. Now, Figure 3a illustrates the situation where a camera moves through multiple stationary objects, fixating at them and calculating each obstacle's looming value.

The camera, while making the translational motion rotates and fixates at each of the four objects $\mathrm{A}, \mathrm{B}, \mathrm{C}$ and $\mathrm{D}$ and the corresponding looming values are calculated. The looming values are plotted in Figure 3b. $L_{A}, L_{B}, L_{C}$ and $L_{D}$ represent the looming values for the objects $A, B$, $\mathrm{C}$ and $\mathrm{D}$ respectively. Note that the looming values of all the four objects are the same at time instant $t_{2}$ since they lie on an equal looming sphere. Also note that at this instant of time the derivative $\mathrm{dL} / \mathrm{dt}$ indicates each curve's rate of change which signifies how close an object is with respect to the translational vector. $\mathrm{dL} / \mathrm{dt}$ is the maximum for $\mathrm{D}$ which is directly on the path of the camera. The graph also shows zero and negative looming values at some time instants.

\section{THE OBSTACLE AVOIDANCE ALGORITHM}

\subsection{The Task :}

The task to be performed is the following :

A camera moving in a constant translational velocity has to reach a visible goal in an environment filled with several obstacles. The camera reaches the goal when the looming value 
of the object exceeds a certain value. The camera is assumed as a point.

\subsection{The Concept :}

The space around the camera is divided into the following three zones depending on two threshold looming values:

- Safety Zone

- Mild Risk Zone

- High Risk Zone

This is illustrated in Figure 4. Note that these zones are moving with the camera and their physical size may expand or shrink depending on the speed of the camera. While moving, the camera pans and fixates at each visible obstacle. The looming value is calculated for each obstacle using the method of relative rate of change of area of the projected image (Section 2.5).

The idea used is that varying values of looming signify varying degrees of threat of collision and higher looming values indicate higher degrees. The looming value of each obstacle is compared with the two threshold values and a risk assignment is performed. Refer to Figure 5. Obstacle A carries a high risk of collision. Similarly, obstacle B and C pose mild and no risk respectively.

The camera needs to alter its course to avert possible collision with an obstacle only if the obstacle lies in the High Risk Zone. While implementing this alteration, the course is refined taking into consideration the obstacles lying in the mild threat zone too. After changing the course, the camera again looks at all the visible obstacles lying in the $\pm 90^{\circ}$ from the instantaneous translational vector and decides the degrees of threat coming from the obstacles. If necessary, it changes its course again. If two objects enter the High Risk Zone at the same time the algorithm takes into account the derivative of the looming value. Figures 6,7 and 8 illustrate these sequences. The camera advances towards the obstacle A until the obstacle enters the High Risk Zone. After changing the course to avoid collision it pans and finds a new path through $\mathrm{B}$ and $\mathrm{C}$. This path is safe. Hence it continues in this direction. The algorithm uses the following notations :

$$
\mathrm{L}_{\text {high }}=\text { High threshold value of the looming. }
$$

For any obstacle if the looming value exceeds this value, then the obstacle lies inside the High Risk Zone and the camera should alter its course to avoid any collision.

$$
\mathrm{L}_{\text {mild }}=\text { Mild threshold value of the looming }\left(\mathrm{L}_{\text {mild }}<\mathrm{L}_{\text {high }}\right) \text {. }
$$

If the looming value for any obstacle is in the range of $\mathrm{L}_{\text {high }}$ and $\mathrm{L}_{\text {mild }}$, then that obstacle is in the Mild Risk Zone. It is not needed to alter the course of the camera, but if it is altering 
anyway because of some high risk obstacle, it should refine its new course so that this obstacle posing mild threat does not become a high risk threat after the camera changes its course.

$\alpha=$ safe passing angle.

This is a clearance angle that the camera should maintain whenever it passes through two obstacles that it is trying to avoid.

\subsection{The Basic Algorithm:}

step 1. Initial Path: A straight line that connects the start and the destination point is the original path. The line along the instantaneous translational motion vector is the current path. Initially the current path and the original path are the same. Start moving along the original path.

step 2. Fixation : Rotate the camera through $\pm 90^{\circ}$ (relative to the current path) fixating at each visible object.

step 3. Calculation of Looming : Using the 'relative rate of expansion' method calculate the looming $\mathrm{L}$ and $\mathrm{dL} / \mathrm{dt}$ of each obstacle.

step 4. Risk Assignment and Course Deviation Decision : Sort the looming values in descending order and compare the values with $\mathrm{L}_{\text {high }}$ and $\mathrm{L}_{\text {mild }}$. If the highest looming value is in the High Risk Zone then alter the course. That obstacle represents the maximum threat. If there are multiple obstacles with the same looming value, then they lie on the equal looming sphere. Look at their time derivative $\mathrm{dL} / \mathrm{dt}$. We consider only positive $\mathrm{dL} / \mathrm{dt}$ since in this case the looming value will increase as opposed to negative value of $\mathrm{dL} / \mathrm{dt}$ where the looming value decreases. The obstacle with the highest $\mathrm{dL} / \mathrm{dt}$ lies closest to the current path and poses the maximum threat. If the highest value of the looming is in the range $\mathrm{L}_{\text {mild }} \leq \mathrm{L}<\mathrm{L}_{\text {high }}$ then it is not necessary to change the course. If the highest value is $L<\mathrm{L}_{\text {mild }}$ then the path is naturally safe.

Hence, if $\mathrm{L}<\mathrm{L}_{\text {high }}$ then go to step 7

else

continue.

step 5. Changing of Heading Vector : Altering the course is necessary to avoid collision with the obstacles inside the High Risk Zone. While altering the course ignore the obstacles lying in the safe zone.

Rotate the camera and fixate at the obstacle posing maximum threat (i.e. maximum looming). Measure this fixating angle from the current path. Also measure the fixating angles 
for other obstacles in the high and mild risk zones. Choose a path that is closest to the current path and maintains the safe passing angle $\alpha$.

step 6. Translation : Move in this altered path for a unit time. Then connect the end of the translational motion vector with the destination by a straight line. This becomes the current path.

step 7. Checking the Destination : If the tip of the translation vector is not within a specified looming value with respect to the destination point, then go to step 2 else the destination has been reached, so stop.

Note : Stay as close as possible to the current path to avoid excessive change in the steering angle.

\section{IMPLEMENTATION}

\subsection{The flight simulator:}

The implementation has been achieved on a miniature, six-degree-of-freedom vision-based flight simulator. The simulator allows autonomous vision-based navigation in a miniature environment. This simulator has been obtained by modifying an IBM Clean Room Gantry Robot (Figure 9). The modification is such that the robot's main controller is not being used, instead it is controlled directly by an 80486 based personal computer that supplies analog signals to the six control loops each corresponding to a velocity input. This new control configuration of the robot allows continuous and smooth motion of the different axes.

The visual input to the computer is obtained from a miniature camera located at the endeffector of the robot. Visual data from this miniature camera is processed in the PC-based vision processor. As a result, pitch, yaw, roll and speed signals are generated and sent to control the robot.

\subsection{Experimental Setup:}

There are several white plastic balls placed in a dark black background as shown in Figure 10. Currently the camera uses only the roll and thus pans only on the horizontal $x-z$ plane. The fixating angle can vary from $90^{\circ}$ positive to $90^{\circ}$ negative relative to the current path. The simulator looks at the sequence of images captured by the camera, continuously changes the fixating angle of it and moves through the white balls by avoiding collisions.

\section{RESULTS}

A threshold has been used to convert the sequence of captured gray level images into binary levels. This simplified the task of identifying the white obstacles. Figures $11 \mathrm{a}$ and $11 \mathrm{~b}$ show the position of the simulator at two time instants. The robot advanced by avoiding the 
obstacles. Figure 12 depicts the obstacles as seen by the camera. As the robot moves towards an obstacle the change of projected area increases prompting it to alter the course when the obstacle enters into the High Risk Zone.

\section{CONCLUSIONS AND FUTURE WORK}

We have presented that visual looming as a cue, together with active fixation of the camera, are useful tools for obstacle avoidance. The results substantiate that hypothesis. Our next phase of work will cover the following areas :

- calculation of looming in more unstructured scenario

- considering the finite size of the camera

- incorporating speed change capabilities of the camera

- using optical flow

- considering moving obstacles

In the actual implementation of our idea we have maintained a somewhat structured environment with white balls in dark background, for ease of locating the obstacles and measuring the looming values. In real world scene naturally the world will not be full of white 3-D balls. However, most of the real world objects and the backgrounds have textures which can be used to calculate the looming values. In that case, separating obstacles from the background and calculating the looming for each of these obstacles become two very crucial and challenging problems.

\section{ACKNOWLEDGEMENT}

The authors would like to thank Prof. P.S. Neelakanta for reviewing this paper and suggesting very useful comments.

\section{REFERENCES}

[1] Aloimonos, J., Weiss, I. and Bandyopadhyay A., "Active Vision". Intl. Journal of Computer Vision, 1, 4, pp. 333-356, 1988.

[2] Aloimonos, J. "Purposive and Qualitative Active Vision", Proc. DARPA Image Understanding Workshop, pp. 816-828, September 1990.

[3] Ballard, D.H., "Animate Vision", Artificial Intelligence, 48, pp. 57-86, 1991.

[4] Raviv, D. and Herman M., "Visual Servoing for Robot Vehicles using Relevant 2-D Image Cues", A book chapter to be published.

[5] Gilbert, E.G. and Johnson, D.W., "Distance Functions and Their Applications to Robot 
Path Planning in the Presence of Obstacles", IEEE J. Robotics Automat., vol. RA-1, No. 1, pp. 21-30, March 1985.

[6] Shin, K.G. and McKay, N.D., "Minimum-time Control of Robotic Manipulators with Geometric Path Constraints", IEEE Trans. Automat. Contr., vol. Ac-30; No. 6, pp. 531-541, June 1985.

[7] Kim, B.K., and Shin, K.G., "Minimum-time Path Planning for Robot Arms and their Dynamics", IEEE Trans. Syst. Man Cybern., vol SMC-15, No. 2, pp. 213-223, March/April 1985.

[8] Khatib, O., "Real-time Obstacle Avoidance for Manipulators and Mobile Robots", The Int., J. Robotics Res., vol. 5, No. 1, 1986.

[9] Hogan, N., " Impedance Control : An Approach to Manipulation", J. Dyn. Syst. Measurement Contr. vol. 107, pp.1-24, March 1985.

[10] Krogh, B.H., "Feedback Obstacle Avoidance Control", 21st Allerton Conf. Commun. Contr. Computing, Urbana, IL, pp. 325-334, October 1983.

[11] -, "A Generalized Potential Field Approach to Obstacle Avoidance Control", Proc. Robotics Int. Robotics Res. Conf., Bethlehem, PA, August 1984.

[12] Newman, W.S. and Hogan N., "High Speed Robot Control and Obstacle Avoidance using Dynamic Potential Functions", Proc. Robotics Automat., IEEE, Raleigh. NC, pp. 14-22, 1987.

[13] Volpe, R. and Khosla, P., "Artificial Potentials with Elliptical Isopotential Contours for Obstacle Avoidance", Proc. 26th Conf. Decision Contr., IEEE. Los Angeles, CA, December 1987.

[14] Nelson, R.C., and Aloimonos, J., "Obstacle Avoidance using Flow Field Divergence", IEEE Trans. on PAMI, 11, No. 10, October 1989.

[15] Alderson, G.J.K., Sully, D.L. and Sully, H.G., "An Operational Analysis of a One Handed Catching Task Using High Speed Photography", J. Motor Behav. 6, pp. 217-226, 1974.

[16] Beek, P.J., "Perception-action Coupling in the Young Infant : An Appraisal of Von Hofsten's Research Program", In M.G. Wade and H.T.A. Whiting (Eds.), Motor Development in Children : Aspects of Coordination and Control, Dordrecht, The Netherlands: MartinusNijhoff, pp. 187-196, 1986.

[17] Bootsma, R.J., The Timing of Rapid Interceptive Actions, Amsterdam: Free University Press, 1988. 
[18] Lee, D.N., "A Theory of Visual Control of Braking Based on Information about Time-tocollision", Perception, 5, pp. 437-459, 1976.

[19] Gibson, J.J., The Ecological Approach to Visual Perception, Cornell University Press, Ithaca, N.Y., 1966.

[20] Raviv, D., "A Quantitative Approach to Looming", National Institute of Standards and Technology Tech. Report, NISTIR-4808, April 1992.

[21] Raviv, D., "Visual looming", Proc., SPIE Conf. on Intel. Robots and Comp. Vision XI : Algorithms, Techniques and Active Vision, Boston, MA, November, 1992.

[22] Horn, B.K.P. and Schunck B.G., "Determining Optical Flow", Artificial Intelligence, 17, pp. 185-204, 1981.

[23] Swain, M.J. and Stricker, M., (Eds.) Promising Directions in Active Vision, NSF Active Vision Workshop, August 1991. 


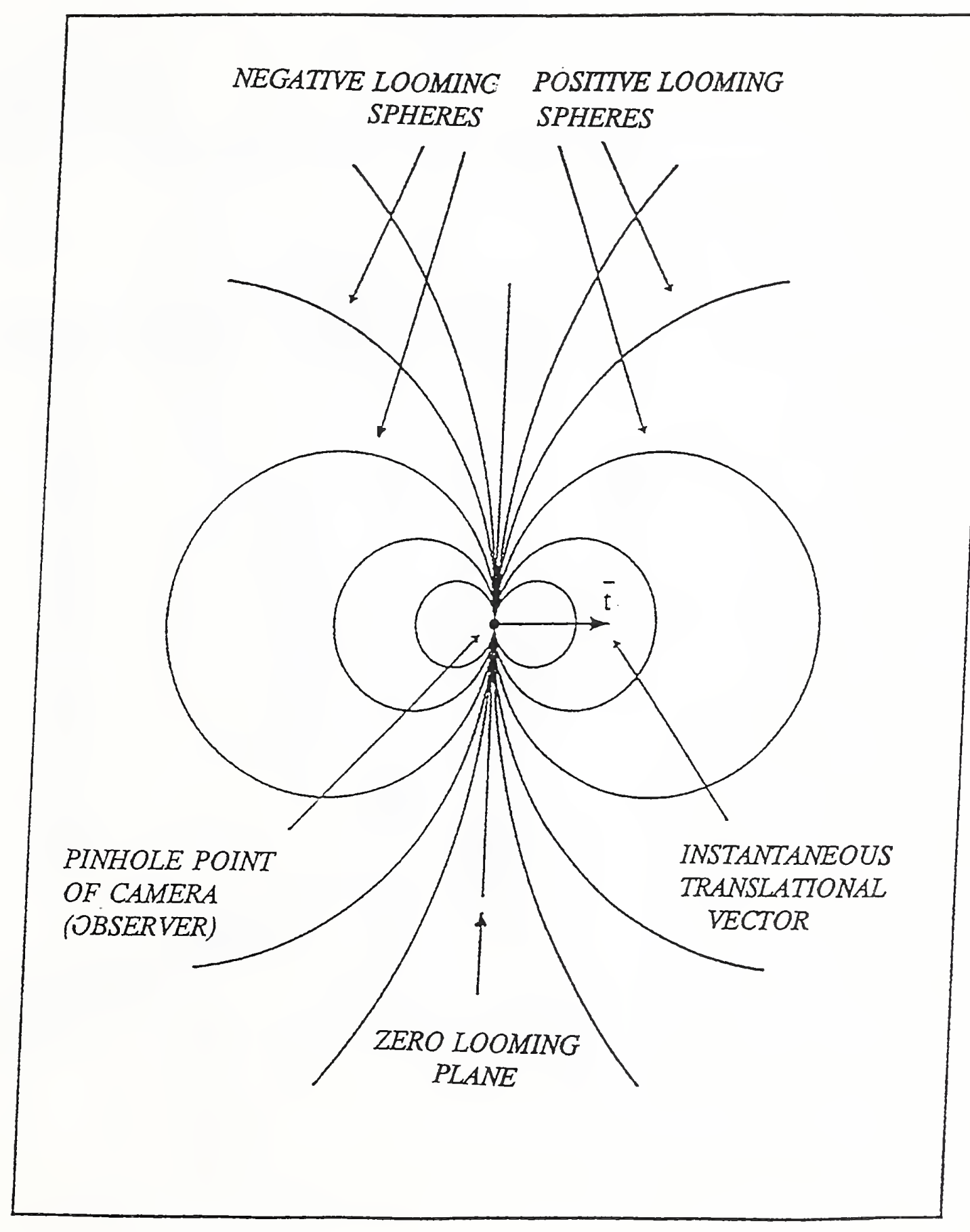

Figure 1: Positive and Negative Looming Spheres and Zero Looming Plane 


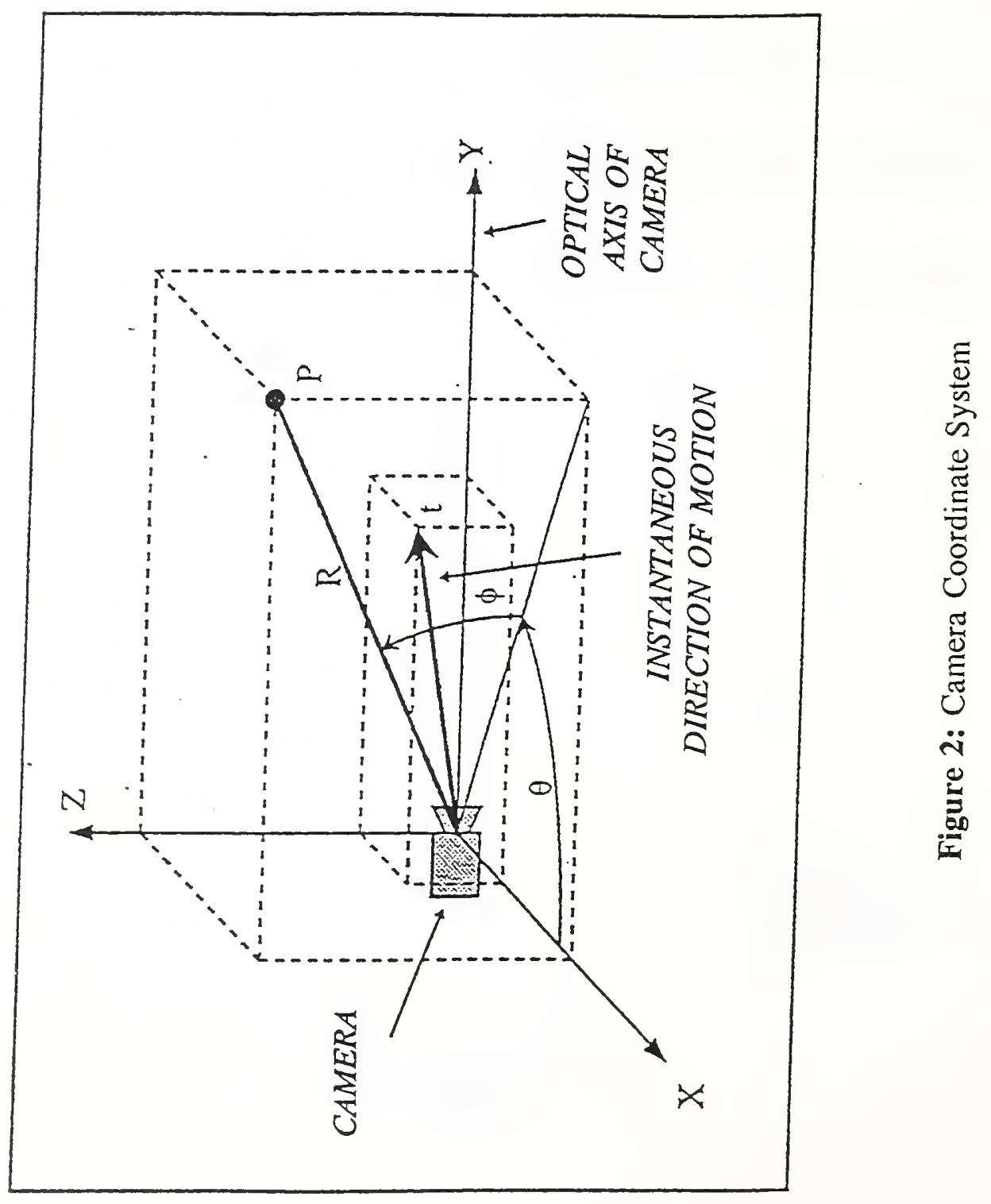




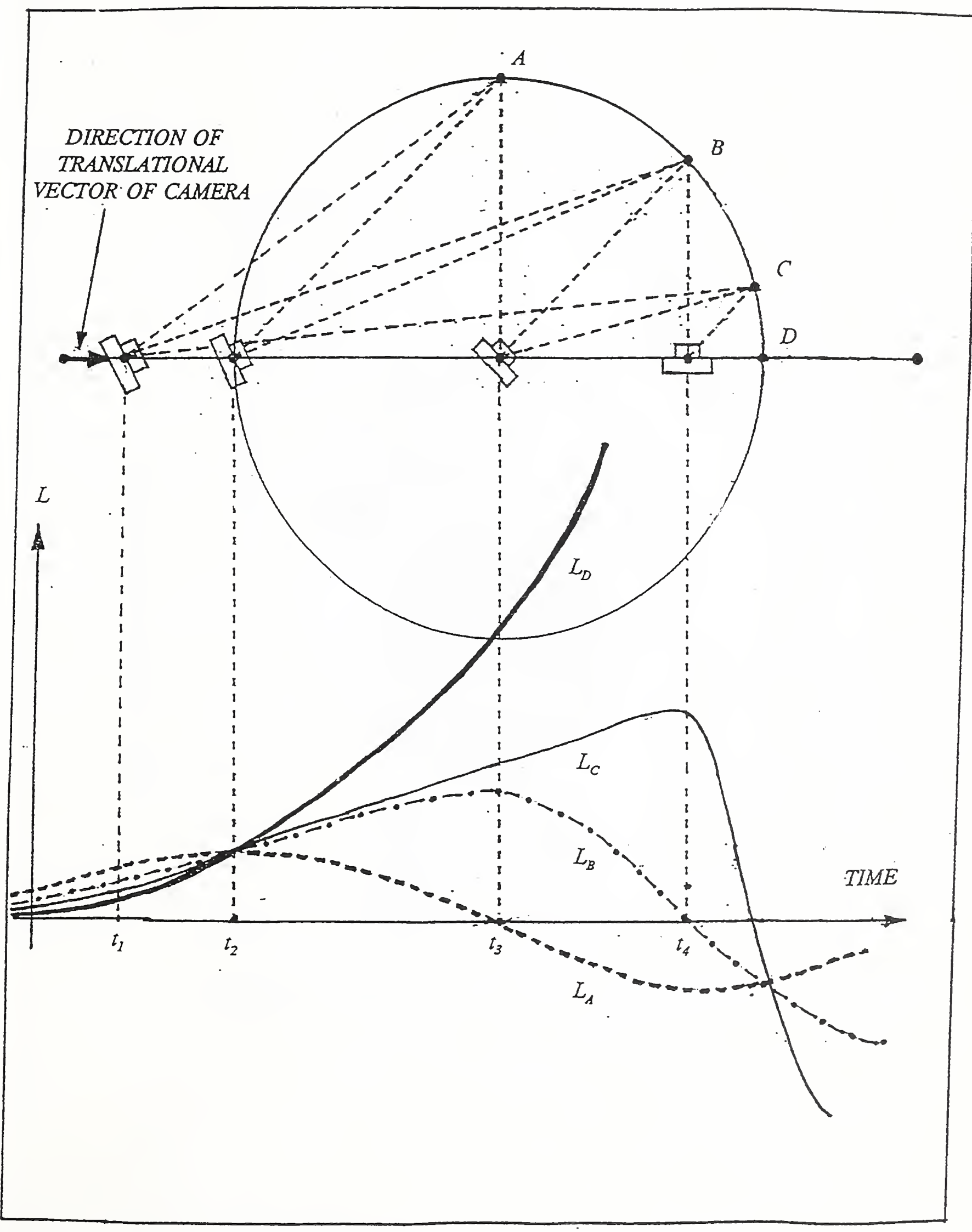

Figure 3: Looming and Fixation as a Function of Time 


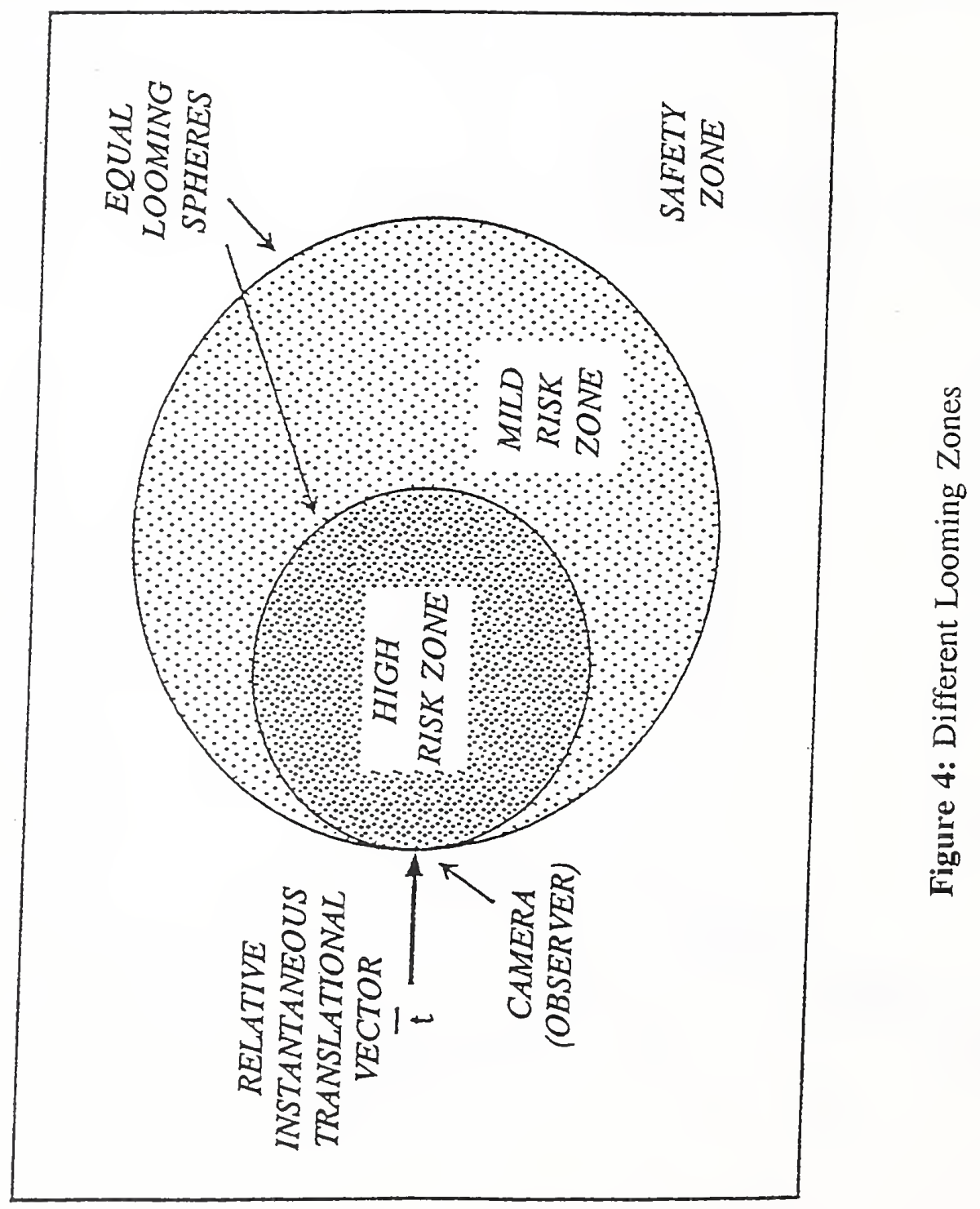




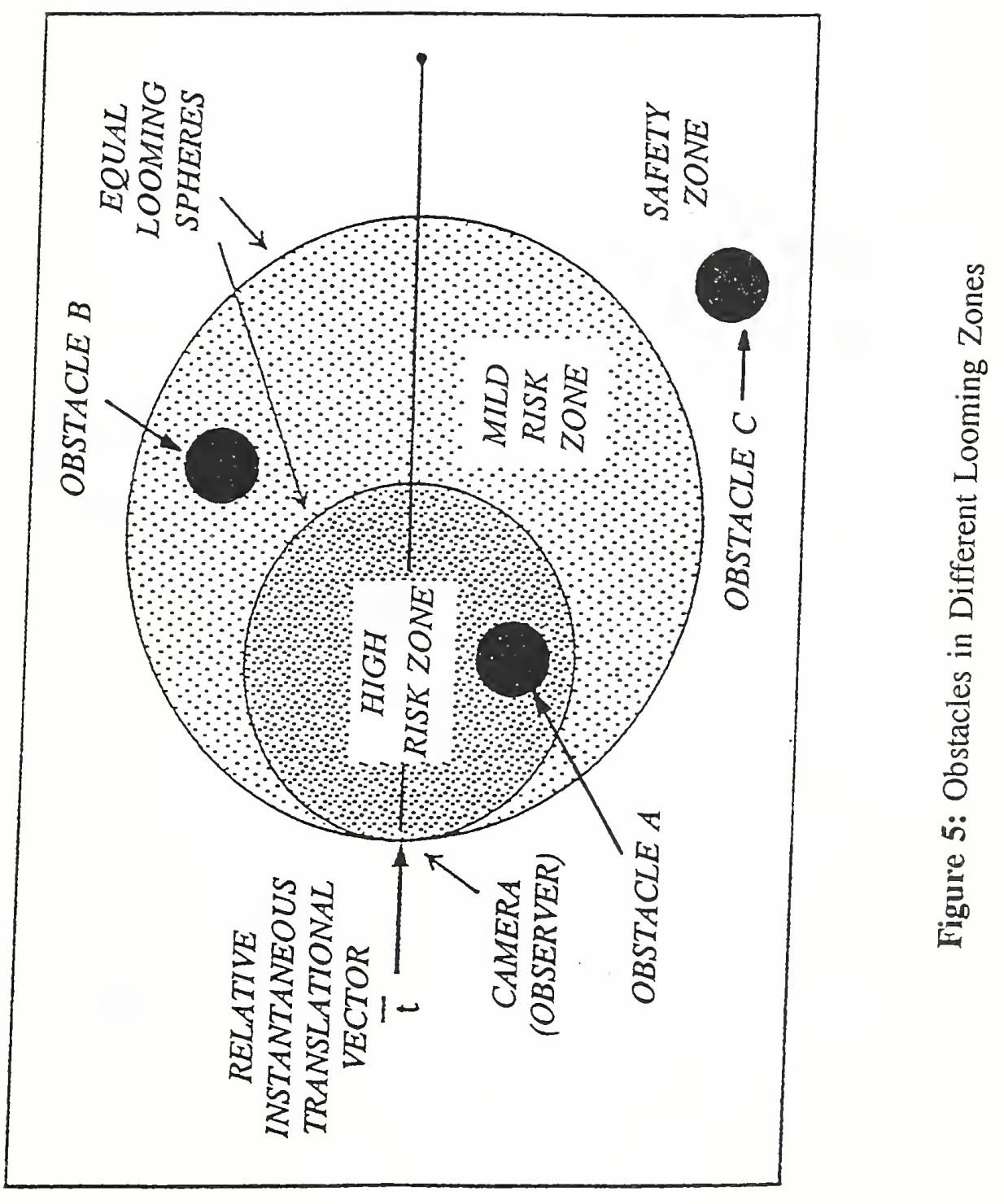




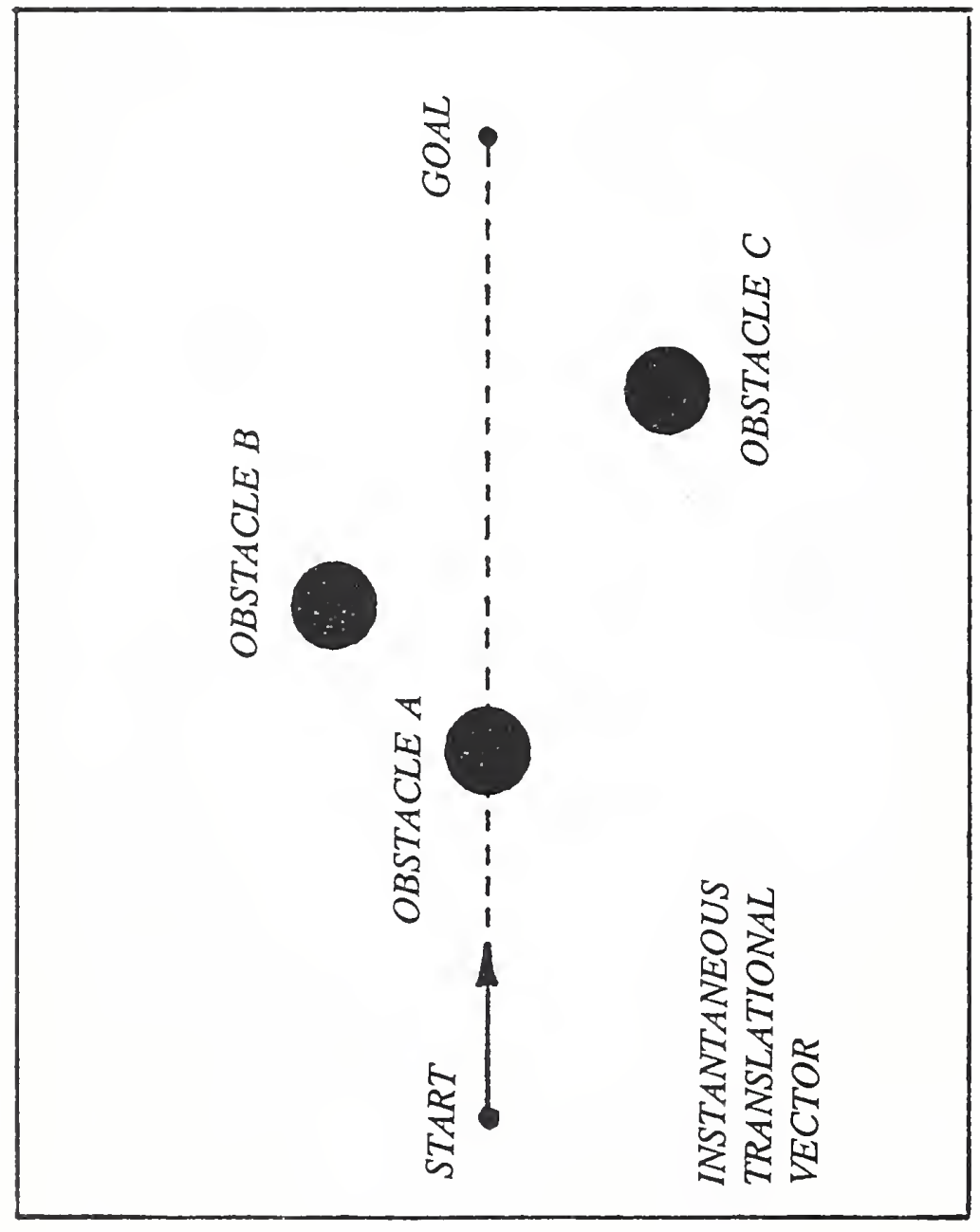

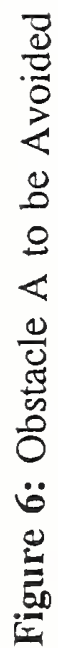




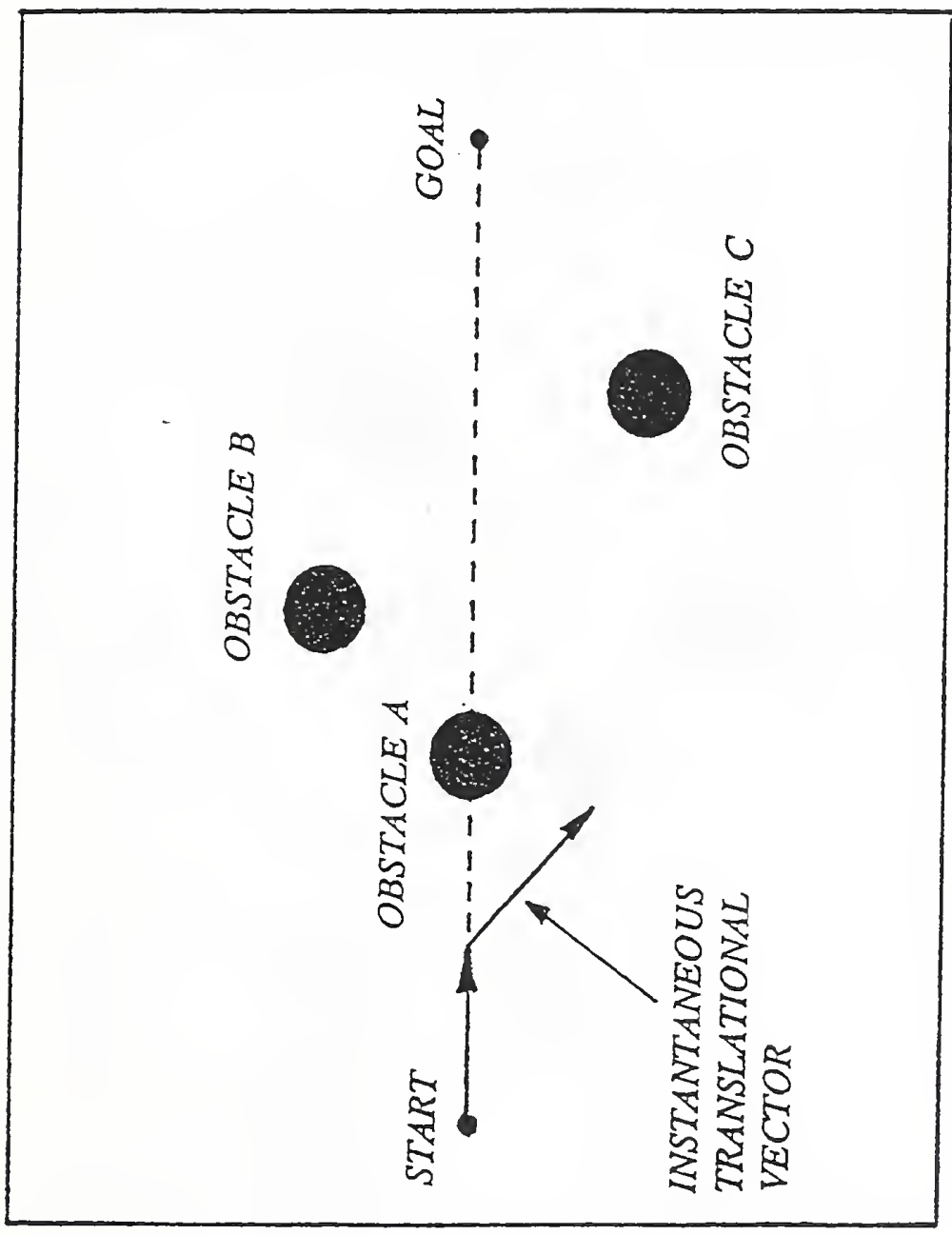

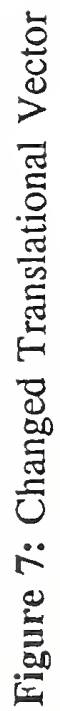




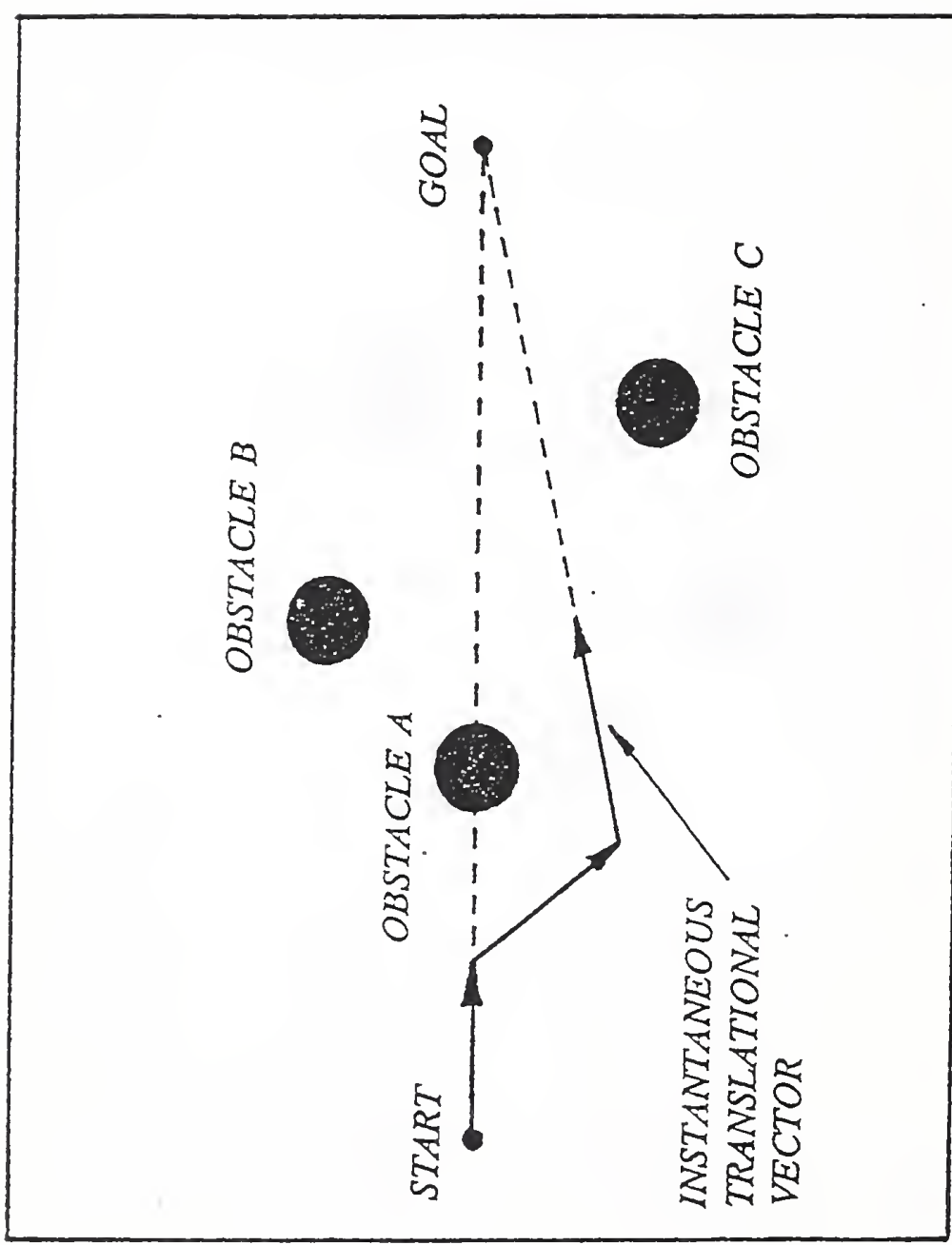

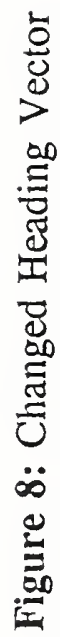




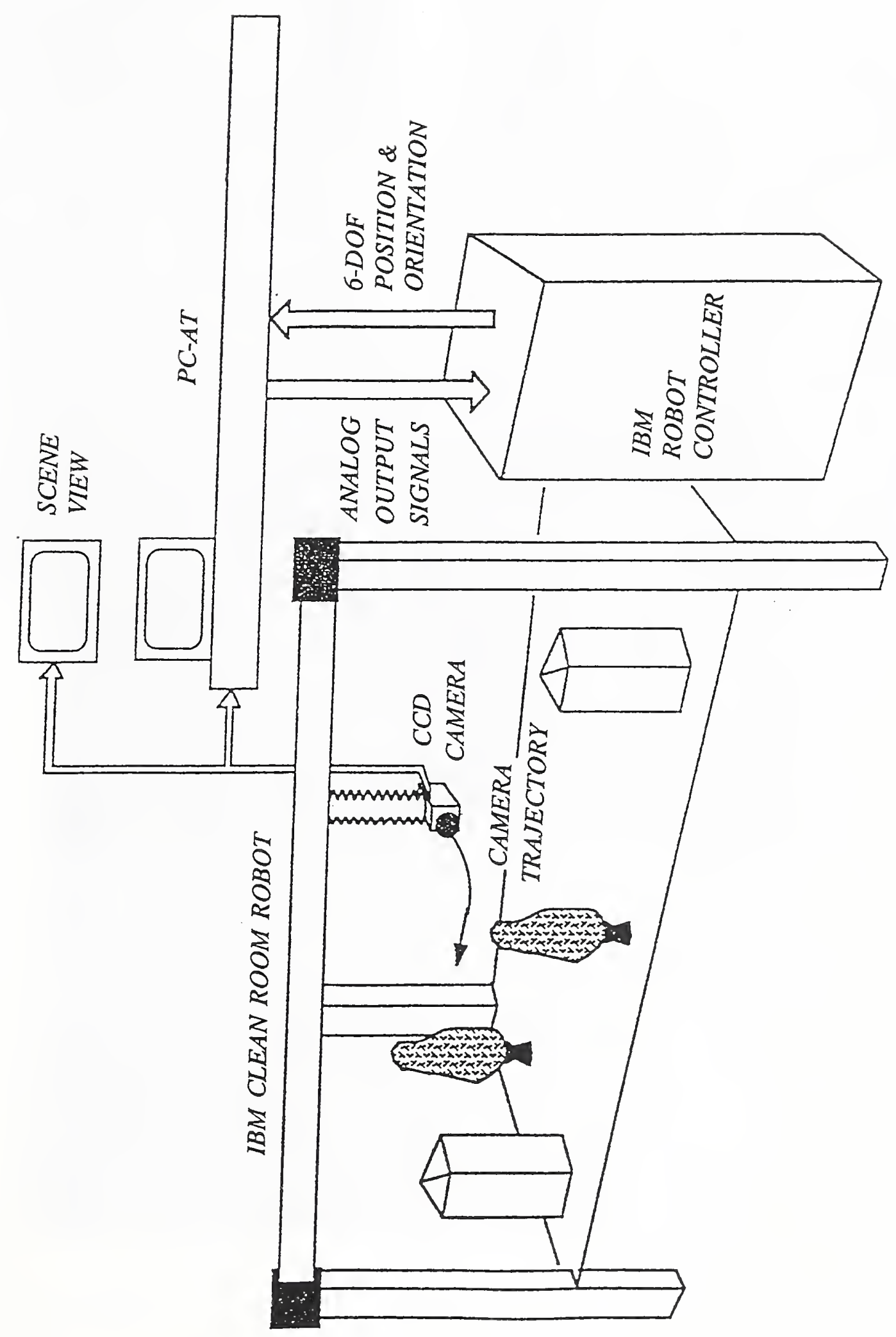

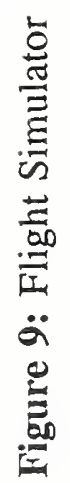




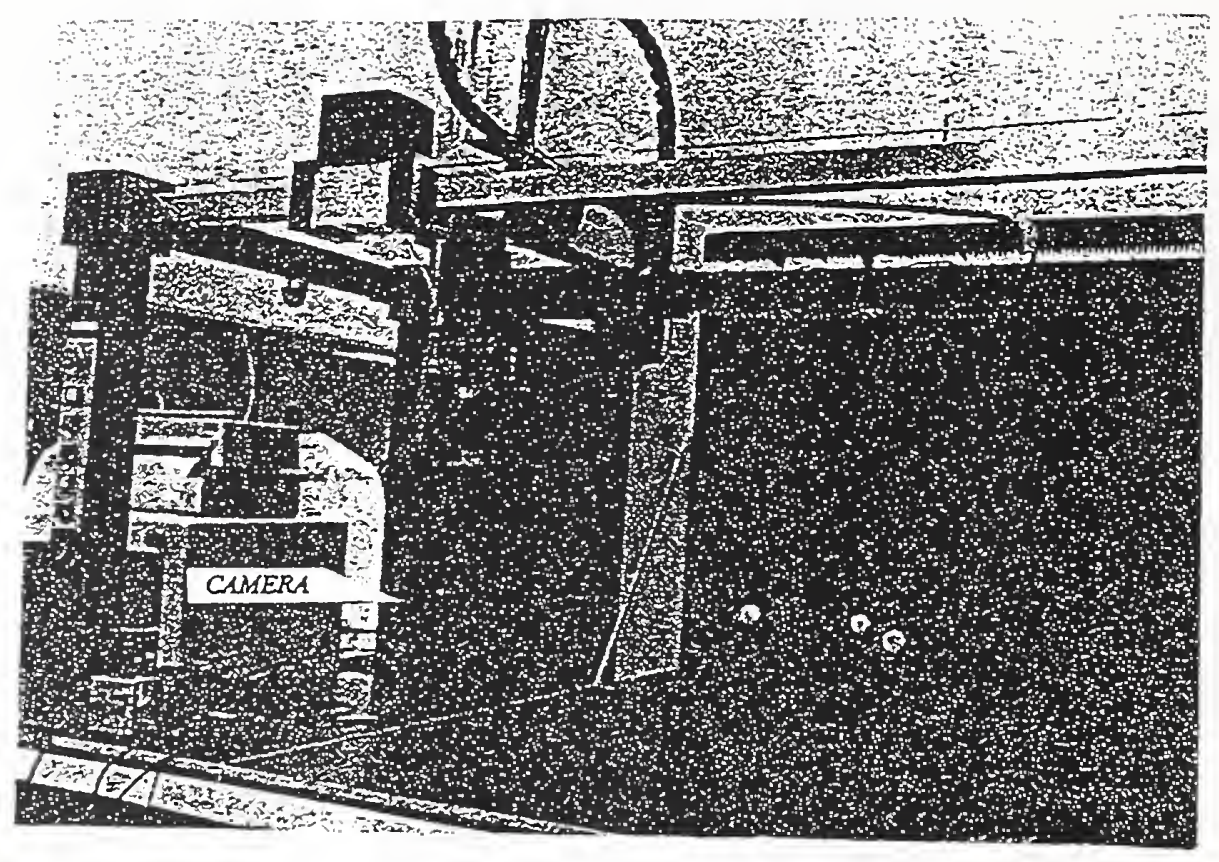

Figure 10: Experimental Setup 


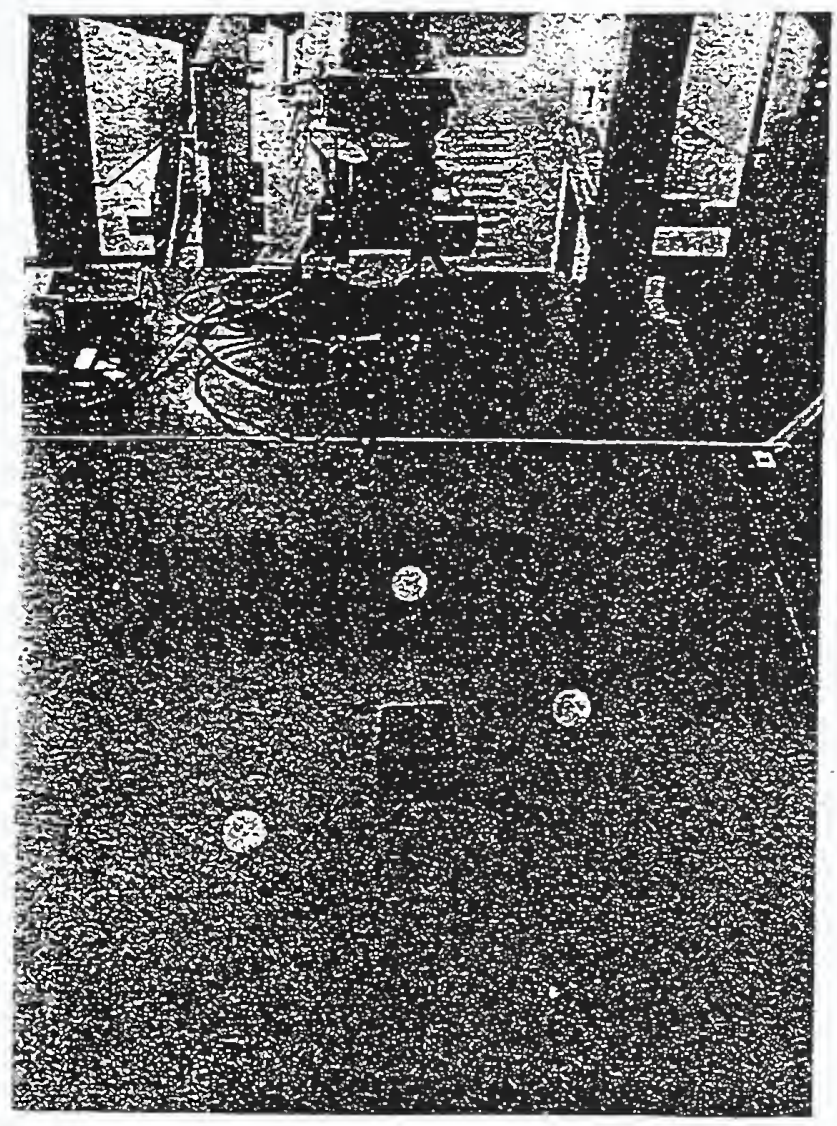

(a) Figure 11: Simulator at Two Time Instants

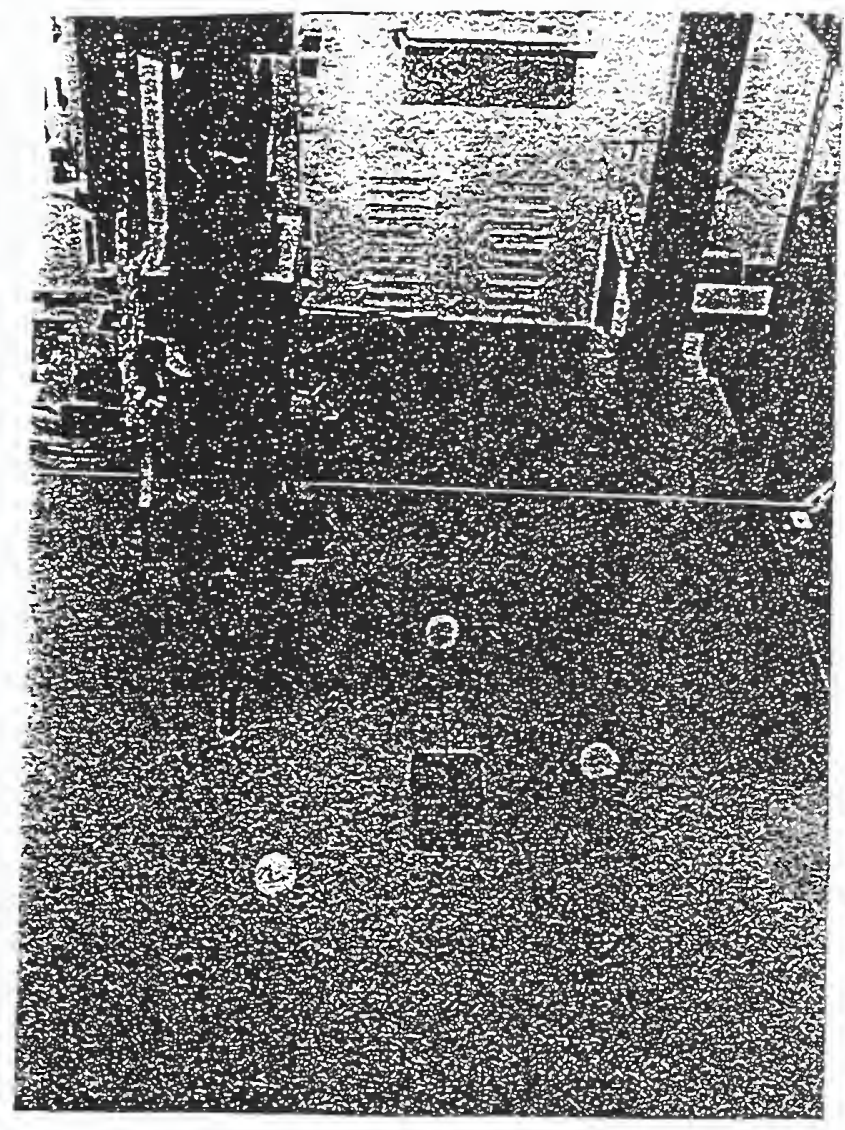

(b)

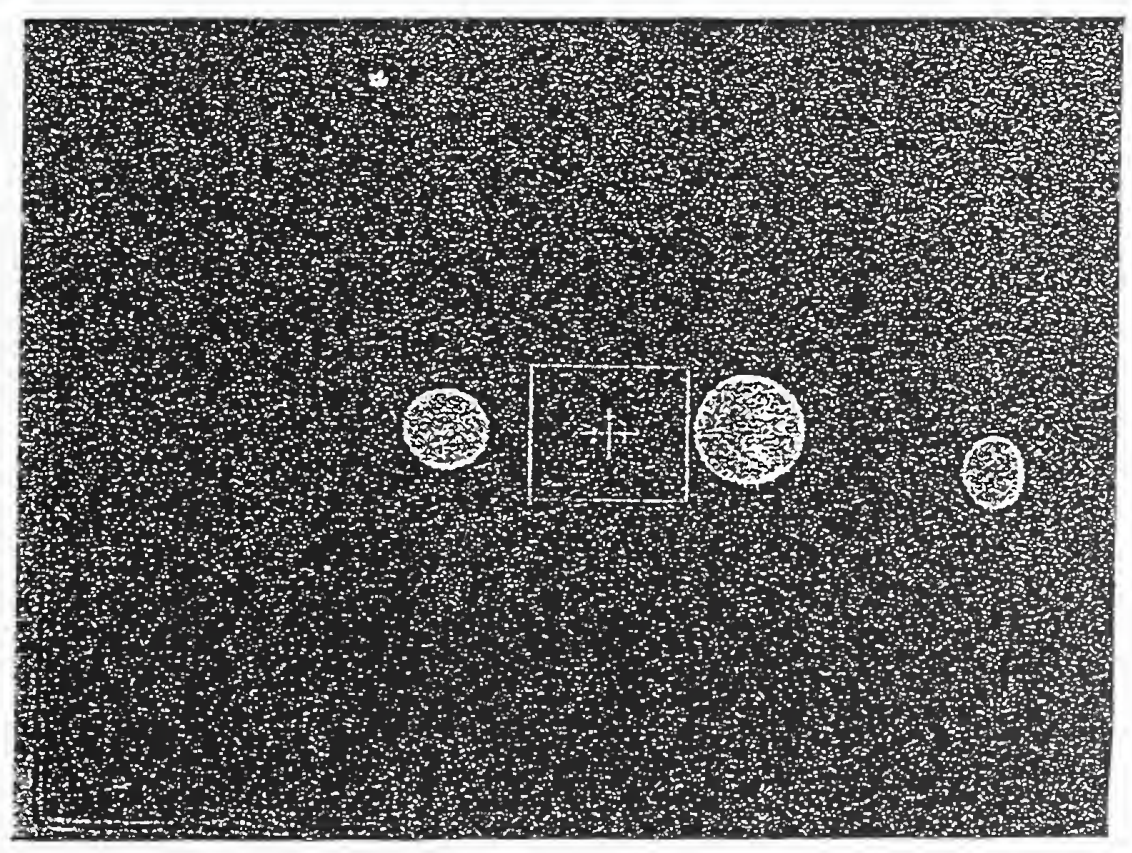

Figure 12: An Image Taken from the Moving Camera 



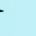

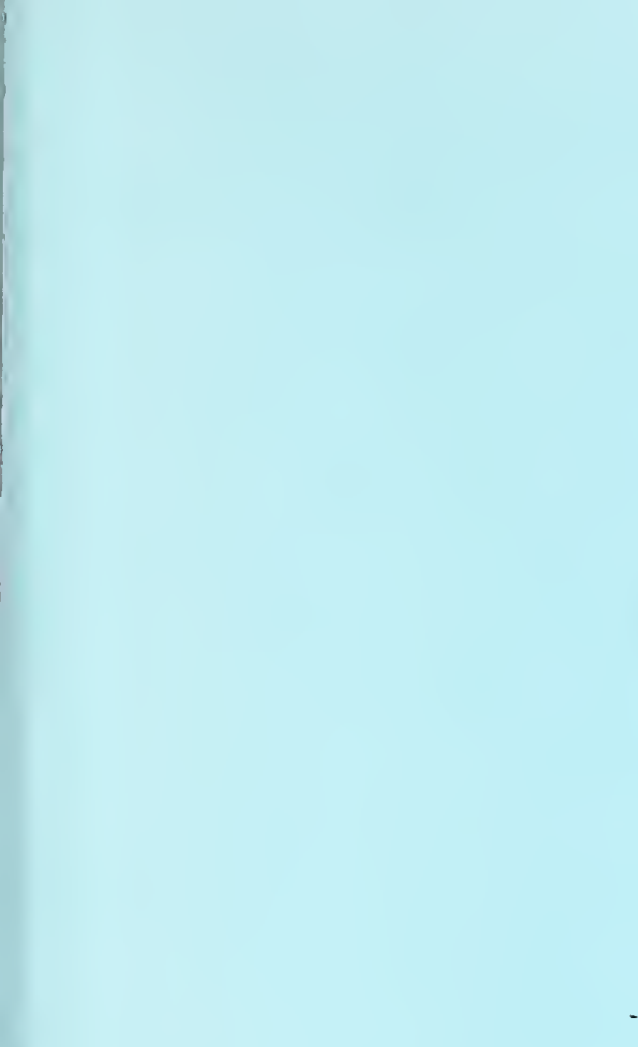

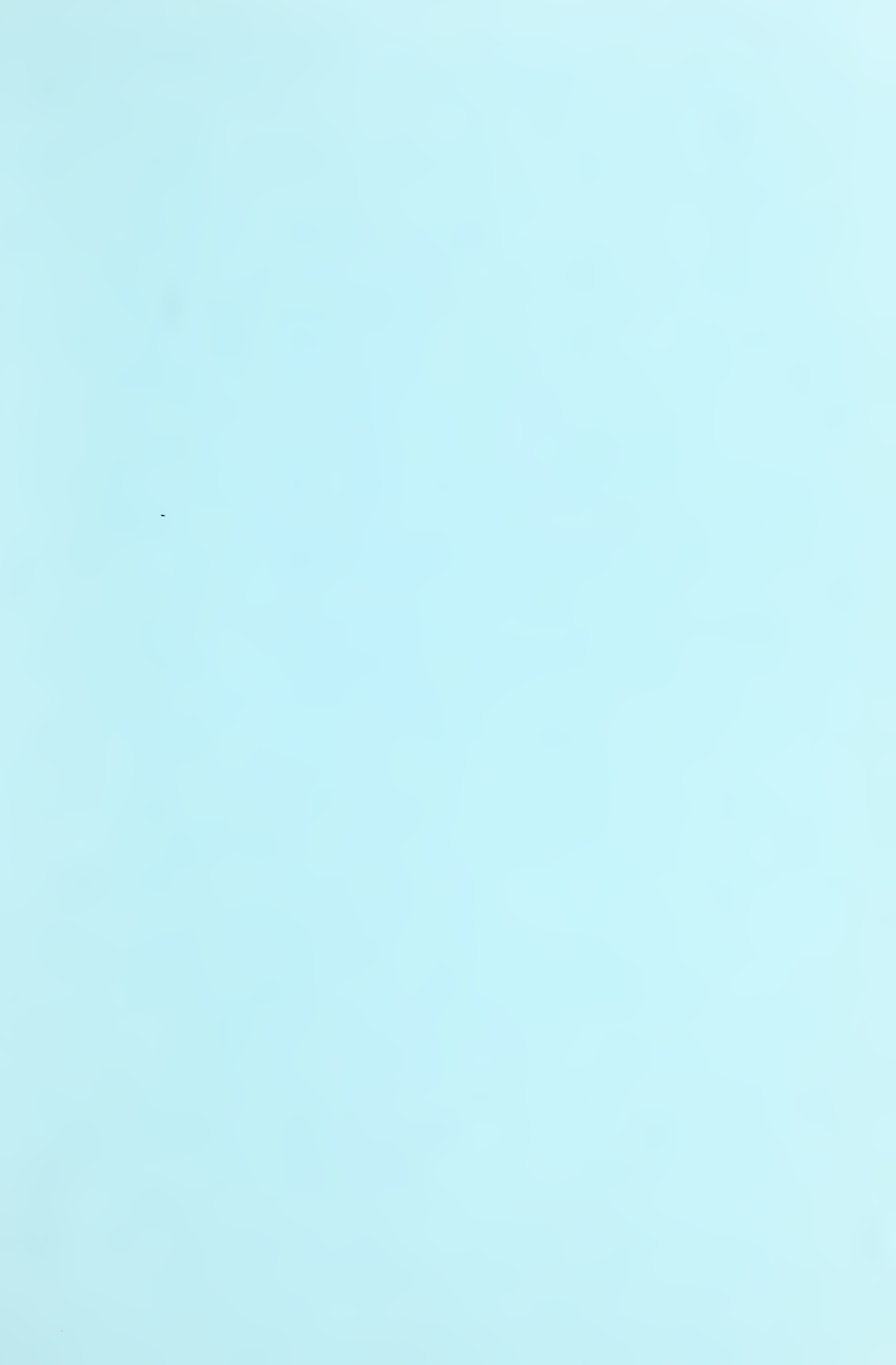

.

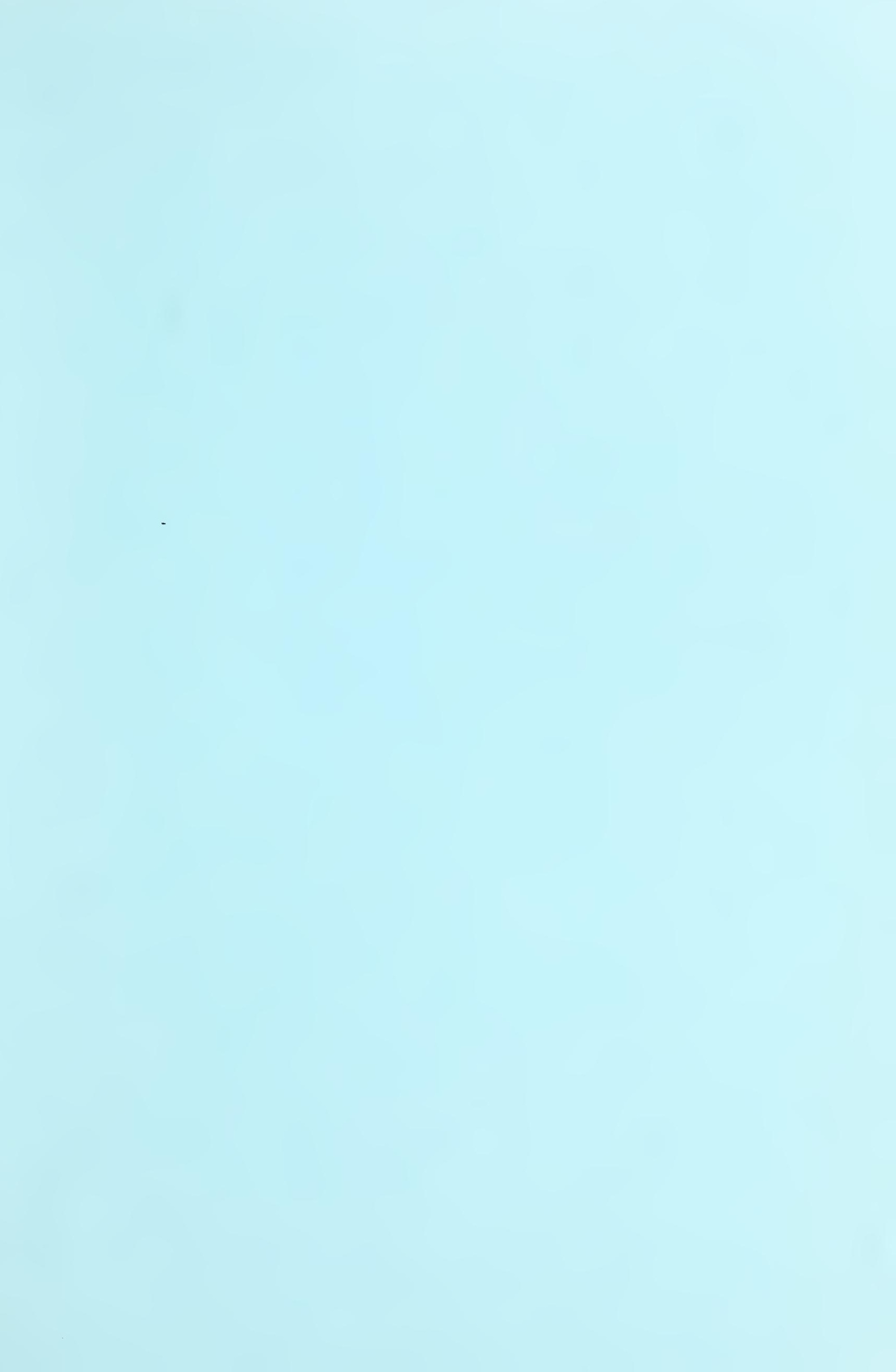


